\title{
Preparation of Oriented Ultrathin Polyethylene Films
}

\author{
Shimpei HASHImoto ${ }^{* 1}$ and Hiroo INOKUCHI ${ }^{* 2, *}$ \\ ${ }^{* 1}$ Research Laboratory, Japan Synthetic Rubber Company, \\ Ikuta, Tama-ku, Kawasaki 214, Japan. \\ *2 The Institute for Solid State Physics, The University of Tokyo, \\ Roppongi, Minato-ku, Tokyo 106, Japan.
}

(Received October 27, 1975)

\section{KEY WORDS Polyethylene / Oriented Ultrathin Film / Cast Film / Stretching / Scanning Electron Micrograph /}

Uniaxially oriented polymer films whose thickness is of tens of $\mu \mathrm{m}$ can be easily obtained by stretching with an ordinary apparatus; such films have been used in the measurement of polarized infrared absorption. ${ }^{1}$ However, when thinner oriented polymer films are required, e.g., less than $100 \mathrm{~nm}$ thick, the usual stretching method is no longer applicable, because the films are too fragile to be stretched successfully.

This report describes a preparative method for oriented ultrathin polyethylene films whose thickness is less than $100 \mathrm{~nm}$, and whose stretching ratio is five or more. These oriented thin films are appropriate for the measurement of anisotropic light absorption ${ }^{2}$ and anisotropic photoelectron emission $^{3}$ in the vacuum ultraviolet wavelength region. Thus, the films are very useful for a study of the electronic structure of the organic polymer and new applications can be expected.

A low density polyethylene (YUKALON MS$30, d_{20}=0.918$ ) was used as the sample material. The polymer was purified by reprecipitation from carbon tetrachloride solution $(12 \mathrm{~g}$ in $500 \mathrm{ml}$, at $65^{\circ} \mathrm{C}$ ), using methanol as a poor solvent. After filtration the precipitated polyethylene powder was kept under vacuum for two days to remove any remaining solvent. Polyethylene $(5 \mathrm{~g})$ purified as above was redissolved in carbon tetrachloride $(500 \mathrm{ml})$ at $65^{\circ} \mathrm{C}$, and, in order to remove any gelatinous material, the solution was filtered under pressure $\left(2 \mathrm{~kg} / \mathrm{cm}^{2}\right)$ through

* Present address: Institute for Molecular Science, Myodaiji, Okazaki 444, Japan. an asbestos filter (pore size, $0.5 \mu \mathrm{m}$ ) at $65^{\circ} \mathrm{C}$. The filtrate solution was kept in a dark place to avoid any photochemical reactions, and used for film-casting.

The preparation of the stretched thin polyethylene films consisted of two steps: in the first step a film having a thickness of about $300 \mathrm{~nm}$ was prepared by the solution casting method, and in the following step, the cast film was stretched up to $400-\%$ elongation. The procedure for film-casting was mainly based on the method described by Carnell, ${ }^{4}$ but as he did not report on polyethylene, the optimum condition for the casting of polyethylene films was determined for the first time. The films were cast reproducibly by withdrawing a glass plate from the polymer solution at a constant rate and temperature, using the apparatus shown in Figure 1. The withdrawal rate was controlled by varying the voltage applied to an induction motor (output, 15W) combined with a reduction gear (ratio, $10: 1)$. By means of a clutch and brake system attached to a winder we could accelerate the withdrawing up to a constant rate, or stop it almost instantaneously.

The glass plate $(35-\mathrm{mm}$ wide, $150-\mathrm{mm}$ long, and $5 \mathrm{~mm}$ thick) made of pyrex glass was pretreated as follows: the surface of the plate was rubbed vigorously with absorbent cotton wetted with water and anionic detergent to remove any coated materials, such as left-over polymer film, and then the detergent was washed off completely with deionized water. After replacing the water by flushing with acetone, the glass plate was immersed in acetone for $30 \mathrm{~min}$ as a 


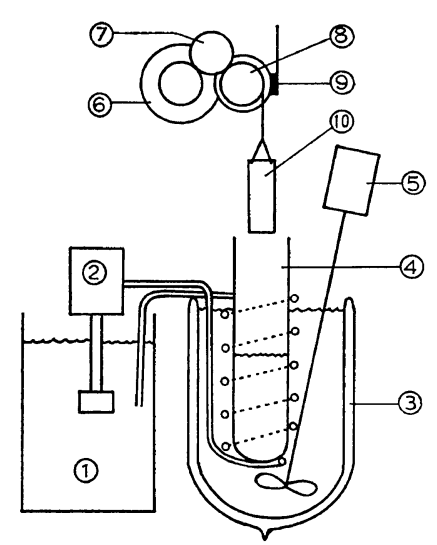

Figure 1. Apparatus for film-casting: 1, thermostat bath; 2, circulation pump; 3, Dewer vessel; 4 , glass testtube; 5 , stirring motor; 6 , geared motor; 7 , clutch; 8 , winder; 9 , brake; 10 , glass plate.

final treatment, according to Carnell's suggestion, ${ }^{4}$ and was allowed to dry in air. The polyethylene carbon tetrachloride solution, placed in a large test tube (60 $\mathrm{mm}$ in diameter and $400 \mathrm{~mm}$ long), was warmed with shaking at $70^{\circ} \mathrm{C}$ in a thermostat bath until the solution became completely transparent. The temperature of the solution was then lowered and maintained at $65^{\circ} \mathrm{C}$. The glass plate was immersed slowly in the polymer solution at the rate of $20 \mathrm{~mm} / \mathrm{sec}$. After $20 \mathrm{~min}$, when thermal equilibrium was attained, all stirring motors were stopped to minimize the mechanical vibrations of the polymer solution and the glass plate was lifted at the rate of 50 $\mathrm{mm} / \mathrm{sec}$. Immediate evaporation of the solvent left a soft polyethylene film on the plate. The thickness of cast films could be controlled by changing the casting condition. The effects of (a) polymer concentration, (b) dipping temperature, and (c) withdrawal rate on the film thickness are shown in Figures $2 a, b$, and $c$. The most significant factor among the casting conditions is polymer concentration, whereas a change in temperature causes only a slight change in the film thickness. The effect of withdrawal rate is also significant, especially in the lower rate region. The film could be removed from the glass surface successfully when the procedure was carried out on the surface of water, as described in the literature. ${ }^{4}$ The thickness of the film thus prepared was estimated to be about
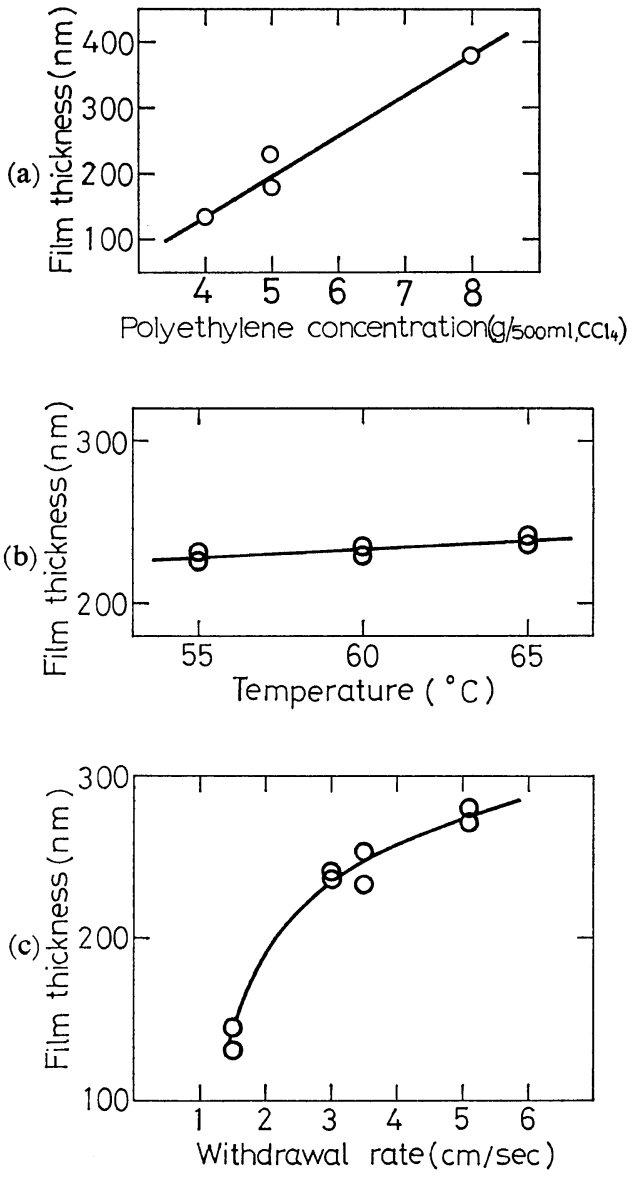

Figure 2. The effect of casting conditions on the film thickness: (a) polyethylene concn, withdrawal rate, $30 \mathrm{~mm} / \mathrm{sec}$ at $60^{\circ} \mathrm{C}$; (b) dipping temp, polymer concn, $5 \mathrm{~g} / 500 \mathrm{ml}$; withdrawal rate, $30 \mathrm{~mm} / \mathrm{sec}$; (c) withdrawal rate, polymer concn, $5 \mathrm{~g} / 500 \mathrm{ml}$ at $65^{\circ} \mathrm{C}$.

$300 \mathrm{~nm}$ by weighing the film of known area after drying it over silica gel for a week. Unevenness in the thickness sometimes occurred when the glass plate was swinging slightly as it was withdrawn from the polymer solution. Such an uneven part of the film, which could be recognized easily by a periodic change of the interference color, was not used because the film would break at that part during the stretching. We also discarded any film having defects like pinholes, which might be produced by some roughness of the glass surface or by other careless treatments. 


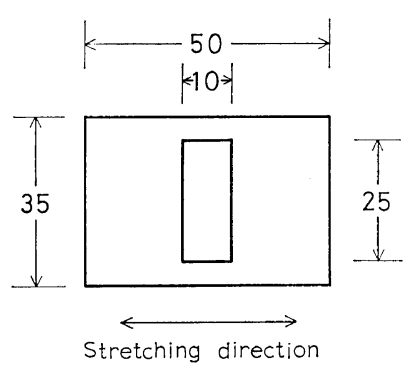

Figure 3. The shape of the rubber frame; the numbers in the figure indicate lengths in $\mathrm{mm}$.

The stretching of such thin cast films could be carried out with the aid of a supporting rubber frame, which was made of a vulcanized styrene-butadiene rubber sheet $1 \mathrm{~mm}$ thick cut to take the shape shown in Figure 3. The stretching was performed as follows. The film floating on the surface of water was cut to an appropriate size (about $40 \times 20 \mathrm{~mm}^{2}$ ) and was picked up on the rubber frame so that the window part of the frame was fully covered by the film, taking care to avoid wrinkles, and then was allowed to dry in air. The thin polyethylene film stuck so fast on the rubber frame that the film did never move on stretching. For stretching, both ends of the frame were held firmly with two metal clamps which were placed parallel to each other and $10 \mathrm{~mm}$ apart, leaving only the window part of the frame free. The clamps then were rapidly drawn apart at room temperature by hand to produce the required elongation. Keeping the elongation by adequate supporters, the stretched film on the frame was rapidly transferred onto an appropriate base. Once mounted, the stretched film was fairly stable, so measurements could be carried out at any later time. The thickness of the stretched films was estimated approximately by dividing the thickness of the cast films by the extension ratio.

The microscopic features of the stretched film of $400-\%$ elongation were observed and photographed (see Figure 4) using a scanning electron microscope. The surface of the film shows distinct necking features: the thicker unstretched parts remain like long islands lying across the oriented fibers of the fully stretched parts. Although the unstretched part is small in area (about $1 / 5$ of the whole surface), the islands seem to act as supports for the film and prevent

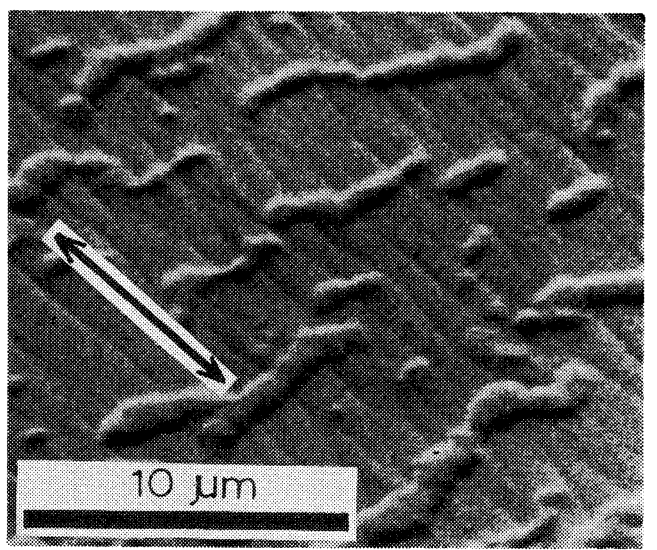

Figure 4. Scanning electron micrograph of a stretched film of $400-\%$ elongation. The arrow indicates stretch direction.

it from splitting. In fact, few split defects could be found on the stretched film up to about $400-\%$ elongation; however, at the higher extensions where the islands would disappear, the film became very fragile and split easily.

One of the difficulties in preparing oriented ultrathin polymer films was the frequent rupture of films during stretching. This failure arose when the cast film had some unevenness in thickness or defects like pinholes. Therefore, a necessary condition for obtaining a uniform and highly elongated film is to start with a uniformly cast film. The uniformity of cast film decreased with decreasing thickness; for instance, the films thinner than about $200 \mathrm{~nm}$ frequently had many pinholes which might have been due to unevenness of the glass surface. Since the effect of the glass surface seemed to diminish with increasing film thickness, we used cast films thicker than about $300 \mathrm{~nm}$ for stretching when highly elongated films were required.

Acknowledgment. The authors wish to thank to Mr. T. Katsuta for taking the scanning electron micrographs.

\section{REFERENCES}

1. For example, D. R. Holmes, R. G. Miller, R. P. Palmer, and C. W. Bunn, Nature, 171, 1104 (1953).

2. The result will be published elsewhere.

3. The result will appear in Chem. Phys. Letters (1976).

4. P. H. Carnell, J. Appl. Polym. Sci., 9, 1863 (1965). 